\title{
Small signal state space model of the frequency-dependent DC cable based on direct vector fitting
}

\author{
S Kovacevic $*$ D Jovcic*, P Rault ${ }^{\dagger}$, A Schwob ${ }^{\dagger}$ \\ * School of Engineering, University of Aberdeen, United Kingdom, r02sk17@abdn.ac.uk, ${ }^{\dagger}$ RTE, France
}

Keywords: Small signal state space model, DC cable, wideband cable model, vector fitting, HVDC.

\begin{abstract}
This paper proposes a new method to represent transmission lines and cables in state space. The method accounts for the frequency dependency of line parameters and essentially translates the state of the art time-domain universal line model into state space. The proposed model is compared against the latest state space model for single DC cable and a bipolar DC cable and it shows certain advantages. DC cables are represented without applying Kron reduction which means that a higher accuracy can be achieved. Single DC cable is represented with a lower model order and higher accuracy for a wide spectre of upper frequency limits and cable lengths. The longer the cable and the higher the upper frequency limit, the more pronounced is the model order reduction. The two state space models are also compared for a $70 \mathrm{~km}$ bipolar DC cable in the frequency range up to $1 \mathrm{kHz}$ and the proposed model achieves higher accuracy with a slightly lower model order. More importantly, the proposed model succeeds at representing the effect of mutual coupling within the desired accuracy criteria. Furthermore, based on the analysis of the two-conductor DC cable (bipolar cable), it is shown that the proposed method overcomes the issue to represent unbalanced multi-conductor lines and cables in state space. The reason is that it avoids modal transformation. Moreover, in case of multi-conductor lines and cables, it is expected that the proposed method is beneficial for longer systems, higher upper frequency limits and for more mutually coupled conductors.
\end{abstract}

\section{Introduction}

State-of-the-art time domain modelling of transmission lines and cables is based on the theory of the universal line model [1]. This model accounts for the frequency dependency of the line parameters and can be used for accurate electromagnetic transient simulations. However, it is not possible to translate it directly into state-space format which is required for system stability studies. Transmission lines and cables have traditionally been represented using single or cascaded PI section model [2]. Although it enables state space representation, this model does not adequately represent the damping and dynamics on the line or cable. In Error! Reference source not found., it is shown that using the PI model can lead to a wrong prediction of HVDC converter- cable interactions modes. To account for the frequency dependency of the transmission lines, it was proposed in [4] to represent longitudinal parameters (inductance and resistance per-unit-length) using multiple parallel RLbranches. This idea was further investigated to assemble state space representation of the frequency dependent transmission line in [5] and the frequency dependent underground DC cable in [3]. The model is essentially an enhanced cascaded PI-section model with multiple parallel RL-branches instead of one. The parameters of the parallel branches are obtained by employing an identification procedure called vector fitting [6] on the frequency response of the series per-unit-length impedance. The model can accurately represent the damping characteristic of a single-conductor frequency dependent transmission line or cable. In case of multi-conductor lines and cables, a modal transformation matrix has to be employed and this matrix is frequency dependent for unbalanced transmission systems. The frequency dependent matrix also has to be translated into state space by the use of the vector fitting and this complicates state space modelling and makes it possibly unfeasible. Furthermore, DC cables present unbalanced multi-conductor cases as each cable is composed of multiple concentric layers. In order to represent DC cables in state space, Kron reduction is typically applied. It assumes ideal grounding for the supplementary layers - sheath and armor and this reduction is justified for small signal HVDC interaction studies [7].

This paper investigates a new method to represent frequency dependent transmission lines and cables in state space. The aim is to eliminate the use of modal transformation matrix in order to enable modelling of unbalanced multi-conductor cases. Such method would also eliminate the necessity to apply Kron reduction in case of DC underground cables. The aim is also to achieve lower model order and higher accuracy for the same upper frequency limit and cable length. The motivation for this comes from the investigation of the resulting high model order of the methodology presented in [3]. The analysis is conducted in [8] and it shows that the model order depends exponentially on the desired frequency range which means the model order can be 100-300 for higher frequency range. For a bipolar cable (two-conductor case), the model order is even twice as large.

The idea proposed here is direct application of the vector fitting method on the frequency responses of the universal line model. The proposed methodology will be compared against the latest state space model [3] in terms of model order and accuracy for different upper frequency limits and different cable length. The two models will be compared for 
single DC cables and for a bipolar DC cable. Based on the investigation of the bipolar DC cable, application of the proposed methodology for multi-conductor transmission systems will be analysed.

\section{Universal line model}

The transmission system models can generally be divided into two main groups: the models with lumped parameters and the models with distributed parameters. The complex behaviour of transmission lines and cables is most accurately represented with the distributed parameter model that also accounts for the frequency dependency of the system. Judging by the availability in commercial electromagnetic timedomain simulation software, the most commonly used example of such model is the universal line model also known as the wideband model [1]. This model is the preferred benchmark model choice and will be used to analyse the performance and accuracy of alternative models in this study. However, this model cannot be translated directly in state space.

\section{State space linear modelling of transmission lines and cables}

\subsection{Single and cascaded PI section model}

The most commonly used model of transmission lines and cables that can be directly translated into state space is the model based on equivalent PI sections [2]. The underlying assumption in derivation of this model is that longitudinal parameters, namely the resistance and inductance per unit length are not frequency dependent. The admittance per unit length can practically be considered as frequency independent because it negligibly changes with frequency. The model is formed by cascading PI sections and the number of sections depends on the length of the line or cable. Although it can be translated directly into state space, this model does not represent the frequency dependent nature of transmission systems and it cannot be used to represent mutual coupling in multi-conductor systems.

\subsection{Cascaded PI section model with multiple parallel ' $R L$ ' branches}

This is the latest state space model of transmission lines and cables [3]. It is an enhanced version of the cascaded PIsection model which represents the frequency dependent longitudinal parameters using multiple parallel ' $R L$ ' branches. The parameters of the parallel branches are obtained by performing curve fitting method called vector fitting [6] on the frequency response of the series per-unitlength impedance. Frequency response is obtained either from universal line model routine or from theoretical formulas. This provides resistances and inductances per-unit-length while RL parameters in the final linear model are calculated considering the length of the line and the number of PI sections. This state space model can accurately represent damping and dynamics of a single-conductor transmission line or cable. The accuracy of the model depends on two parameters: the number of cascaded PI sections and the number of parallel RL branches. The shortcoming of this model is that the model order depends exponentially on the desired frequency range and this is particularly pronounced for longer cables. The reason for this is that longer cables require more PI section and each section can have several elements.

Representing a multi-conductor system requires modal transformation which is based on the well documented theory of eigenvectors. In the case of unbalanced systems (conductors having different impedances with respect to ground), modal transformation matrix is frequency dependent. This makes deriving state space model difficult and perhaps not feasible. The reason is that the transformation matrix also has to be translated into state space using vector fitting.

DC cables are typically composed of multiple concentric conductor layers such as conductor, sheath and armor. This means they present unbalanced multi-conductor systems. In order to derive state space model, Kron reduction is applied to reduce the cable to a single-conductor system thus avoiding modal transformation. Kron reduction assumes ideal grounding of the supplementary layers (sheath and armor) along the entire cable length. In reality, sheath and armor are grounded at the cable ends or at several points along the cable, however, Kron reduction is justified for small signal HVDC interaction studies [7].

\section{State space model based on direct vector fitting}

A new state space model of transmission lines and cables is proposed. The model is derived by translating the timedomain universal line model into state space. Universal line model is regarded as a 'black box' model and frequency scan is performed from every input to every output. Then, vector fitting is applied to translate the obtained frequency responses into a multi-input multi-output state space model. The proposed approach is said to be based on direct vector fitting because it directly translates the universal line model into state space without employing modal transformation or forming cascaded PI sections. The latest state space model from section 3.2 is derived by obtaining frequency response of the series per-unit-length impedance while the proposed method is derived by performing frequency scan directly on the transmission line or cable.

\subsection{Direct vector fitting procedure}

The proposed method requires a frequency scan from each input to each output of the universal line model in timedomain software. It can be performed either by applying AC voltage at the desired input and then by measuring AC current at the desired output or the other way around. This determines whether the final model will have voltages or current as the inputs or outputs. The frequency responses are obtained using logarithmically spaced frequencies within the frequency range of interest. The reason for this is that linearly spaced 
frequency response requires significantly more frequency samples which makes frequency scan impractical to perform. Logarithmically spaced response has a higher density of frequency samples at lower frequencies so vector fitting will naturally put focus on the fitting accuracy in this range. However, vector fitting also has a weighting feature that can set focus of the fitting accuracy on an arbitrary frequency range [3][9]. This means that it can counteract the fact that the frequency samples are logarithmically spaced in order to achieve more balanced fitting accuracy. The idea is to use a logarithmically spaced vector as the weighting function which is tuned by trial and error.

Vector fitting is capable of fitting multiple frequency responses stacked in a column using the same set of poles [9]. This means that it can automatically derive state space representation of a single-input multi-output system. The final multi-input multi-output system is formed by combining matrices obtained for individual inputs. The matrices are combined in the following way:

$$
\begin{aligned}
A & =\left[\begin{array}{ccc}
A_{1} & 0 & 0 \\
0 & \ddots & 0 \\
0 & 0 & A_{N}
\end{array}\right] \\
B & =\left[\begin{array}{ccc}
B_{1} & 0 & 0 \\
0 & \ddots & 0 \\
0 & 0 & B_{N}
\end{array}\right] \\
C & =\left[\begin{array}{lll}
C_{1} & \cdots & C_{N}
\end{array}\right]
\end{aligned}
$$

where $\mathrm{A}, \mathrm{B}, \mathrm{C}$ are the total state space matrix, input matrix and output matrix respectively. $\mathrm{A} i, \mathrm{~B} i$ and $\mathrm{C} i$ are the matrices for input $i$, with $\mathrm{N}$ being the total number of inputs. So, the final model order is determined by the number of inputs and the fitting order of single-input multi-output systems.

\subsection{Advantages of the proposed methodology}

The advantages of regarding the transmission system model as a black box is that there is no need for modal transformation. This overcomes the issue to represent unbalanced systems that arises when using the latest approach from section 3.2. This also means that DC cables can be represented without applying Kron reduction which suggests that improved model fidelity and higher accuracy might be achieved. When the Kron reduction is not applied the cable has multiple inputs corresponding to all the cable layers. However, the cable can still be regarded as a black box system with the number of inputs equal to the number of basic conductors. The reason for this convenience is that the sheath and armor inputs are always at ground potential. This is exhibited in Figure 4.1 for a common bipolar DC cable.

Since the proposed model is not formulated using PI section, it is suspected that the model order will not depend exponentially on desired frequency range. Next section thoroughly investigates model order and accuracy for single DC cables and for a $70 \mathrm{~km}$ bipolar DC cable.

Black box model

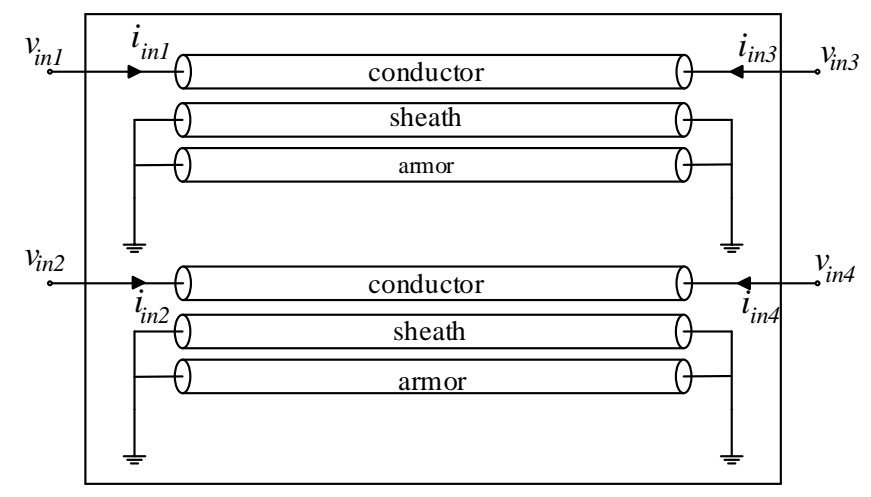

Figure 4.1 Schematic representation of a bipolar DC cable regarded as a 'black box' model

\section{State space model simulations}

\subsection{Performance indices}

The proposed model is compared against the latest state space model to verify theoretical approach and analyse possible advantages. Universal line model in EMTP is used as benchmark model to evaluate performance of state space models. The accuracy of state space models is assessed in frequency domain by the means of the maximum magnitude error and the maximum phase errors. This study is oriented towards providing state space cable model for HVDC stability assessment. Therefore, maximum error is more relevant as opposed to the average error over the frequency range of interest. The reason for this is that in stability studies, a high error in a single frequency cannot be accepted even if the average error is small [3]. The maximum magnitude and phase errors are assessed separately and they are defined by:

$$
\begin{gathered}
\varepsilon_{\text {mag }}=\max _{w \leq w_{B}} 20 \cdot \log _{10}\left|\frac{Z_{f i t}(w)}{Z(w)}\right| \\
\varepsilon_{\text {ang }}=\max _{w \leq w_{B}} 20 \cdot \log _{10}\left|\angle Z_{f i t}(w)-\angle Z(w)\right|
\end{gathered}
$$

where $\mathrm{Z}_{\text {fit }}$ and $\mathrm{Z}$ are the impedance of the state space and the wideband cable model respectively. $\mathrm{w}_{\mathrm{B}}$ is the angular frequency corresponding to the upper frequency limit and $\varepsilon_{\text {mag }}$ and $\varepsilon_{\text {ang }}$ are the maximum magnitude and phase error respectively. Furthermore, the termination impedance of the cable influences the model accuracy. The case of the shortcircuited cable termination is used for the analysis because it provides larger error in comparison to the other extreme termination case, i.e. the open ended cable.

\subsection{Universal line model - Kron reduction}

The proposed method avoids the use of Kron reduction for DC cables. However, Kron reduction is justified according to [7] for small signal HVDC interaction studies. So, it is interesting to investigate the influence of this assumption as 
this could be initial indicator of the accuracy of the proposed model.

A $300 \mathrm{~km}$ DC cable is represented in EMTP using the universal line model. The model is assembled with and without applying Kron reduction. Figure 5.1 compares frequency responses of the universal line model with shortcircuited termination for the two cases. As can be seen from the figure, the maximum difference is perhaps few percent. Although this might not be significant it shows that a higher accuracy can be achieved using the proposed method. The next step is to thoroughly analyse minimum model order against the desired accuracy.
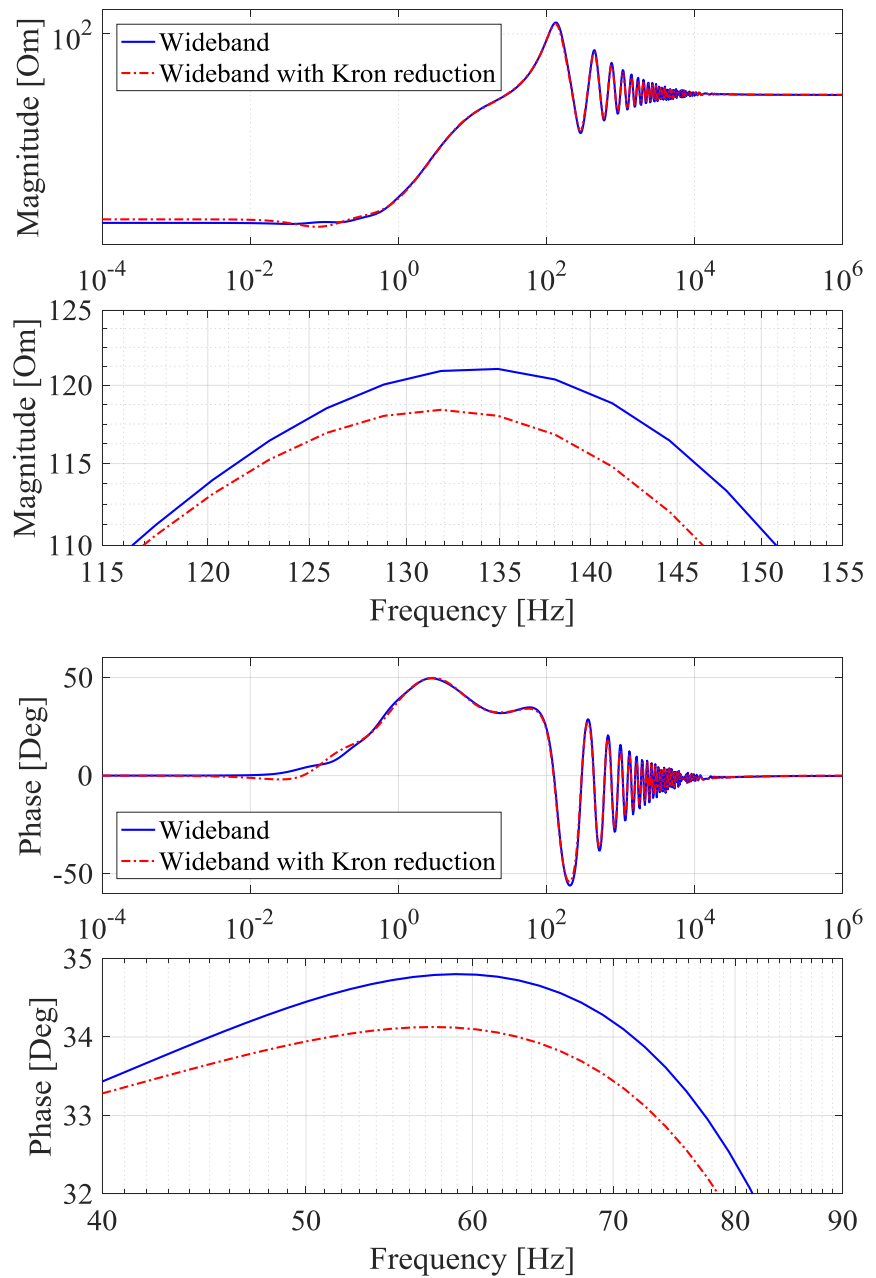

Figure 5.1 Frequency response of the wideband cable model with and without Kron reduction. Figures from top to bottom: magnitude, zoomed magnitude, phase and zoomed phase.

\subsection{Single-conductor DC cable - model order analysis}

The proposed method is compared against the latest state space model from section 3.2 for single-conductor DC cables. The models are compared in terms of minimum model order for various upper frequency limits and cable lengths. Also, two sets of criteria are established for the accuracy. The first is maximum magnitude error of $1 \mathrm{~dB}$ and maximum phase error of $15^{\circ}$ and the second, more stringent, is maximum magnitude error of $0.1 \mathrm{~dB}$ and maximum phase error of $1^{\circ}$. The latest state space model requires employing Kron reduction. The proposed model avoids this assumption and it is assembled by first performing frequency scan of the universal line model in EMTP. This model is regarded as a two-input two-output black box model and frequency scan is performed only from one input to both outputs. The reason for this convenience is that the single-conductor cable is symmetrical in terms of the transfer functions. The low limit for the frequency scan is $0.01 \mathrm{~Hz}$ because the cable impedance changes negligibly below this frequency. Also, it is impractical to perform frequency scan in this range (it requires $10^{3}$ order of the simulation time in seconds). The two obtained frequency responses are translated into a singleinput two-output state space model using vector fitting [9] and then by combining the matrices as described in section 4.1 the final two-input two-output model is obtained. The challenge that arises is to tune the vector fitting weighting function of two responses with the aim of minimizing model order.

Figure 5.2 displays the achieved minimum model order of the proposed state space model while Figure 5.3 displays the results for the more stringent criteria. These results are compared against the results for the latest state space model [8] as shown in Table 5.1 and Table 5.2

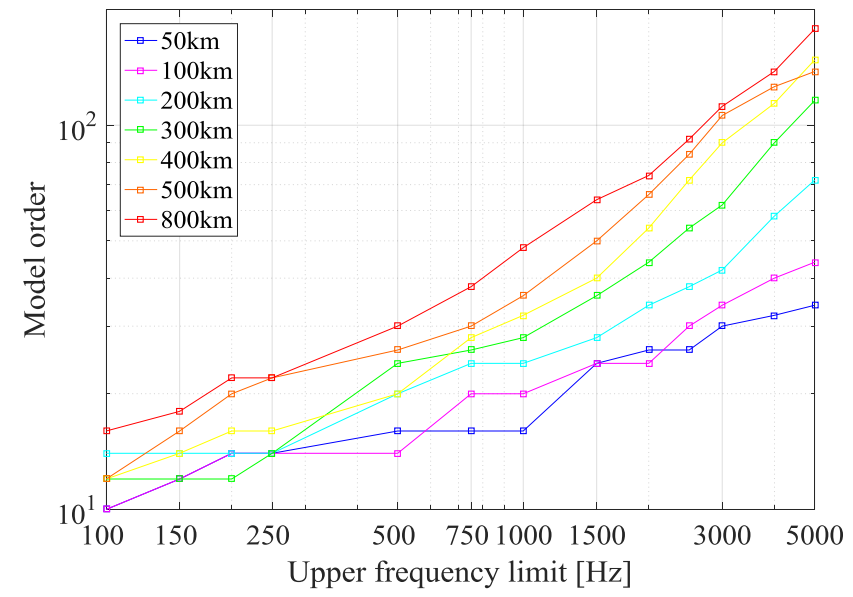

Figure 5.2 Minimum model order for maximum magnitude error $1 \mathrm{~dB}$ and phase error $15^{\circ}$

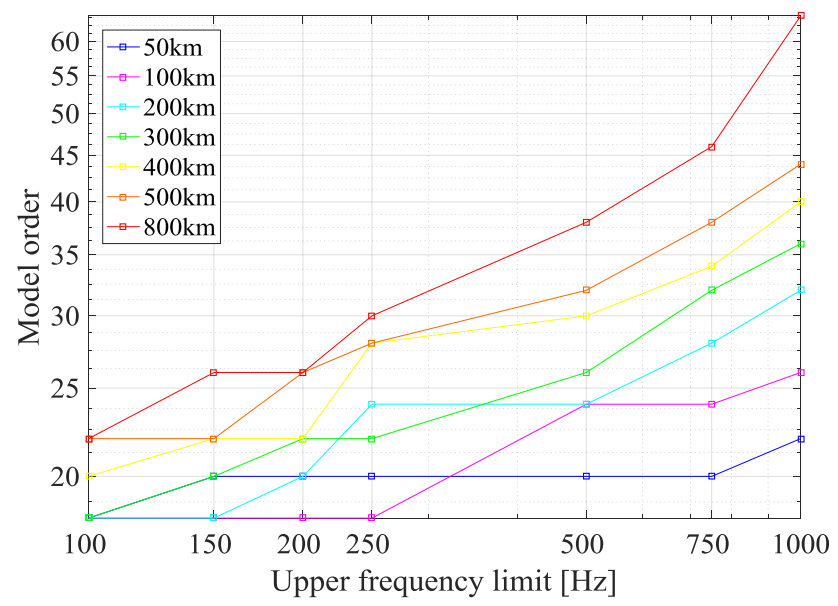

Figure 5.3 Minimum model order for maximum magnitude error $0.1 \mathrm{~dB}$ and phase error $1^{\circ}$ 
Table 5.1 Modelling method that gives lower model order for maximum magnitude error $1 \mathrm{~dB}$ and phase error $15^{\circ}$.

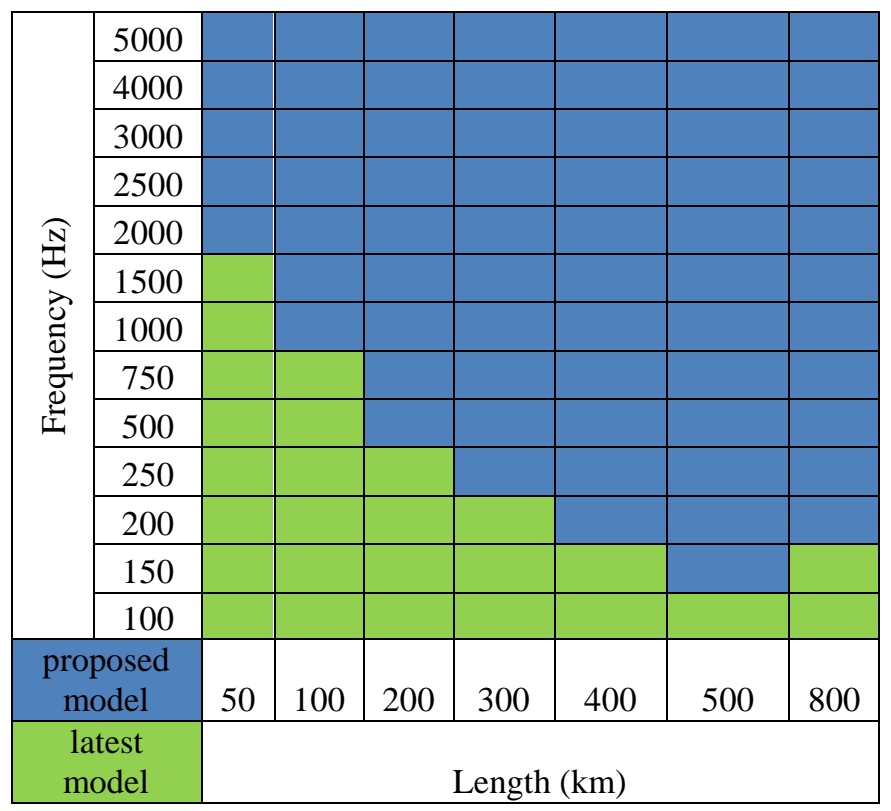

Table 5.2 Modelling method that gives lower model order for maximum magnitude error $0.1 \mathrm{~dB}$ and phase error $1^{\circ}$.

\begin{tabular}{|c|c|c|c|c|c|c|c|c|}
\hline \multirow{7}{*}{ 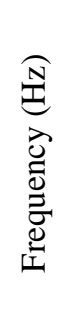 } & 1000 & & & & & & & \\
\hline & 750 & & & & & & & \\
\hline & 500 & & & & & & & \\
\hline & 250 & & & & & & & \\
\hline & 200 & & & & & & & \\
\hline & 150 & & & & & & & \\
\hline & 100 & & & & & & & \\
\hline & $\begin{array}{l}\text { osed } \\
\text { del }\end{array}$ & 50 & 100 & 200 & 300 & 400 & 500 & 800 \\
\hline & & & & & engt & (m) & & \\
\hline
\end{tabular}

According to the conducted analysis for the single-conductor DC cable it can be concluded that:

- Proposed method achieves lower model order and higher accuracy for longer cables and higher upper frequency limits.

- Model order of the proposed method does not depend exponentially on the upper frequency limit. This means significant model order reduction for higher upper frequency limits.

- The difference between the minimum model order of compared methods is more pronounced for the more stringent error criteria. The proposed method achieves higher accuracy with the lesser increase of the model order.

- For lower frequency range (approximately below $250 \mathrm{~Hz}$ ) and/or shorter cables, the proposed approach might not be beneficial.
Table 5.3 provides model order results for some cable lengths and upper frequency limits.

Table 5.3 Minimum model order of two state space models for some cable length and the two accuracy criteria

\begin{tabular}{|c|c|c|}
\hline $\begin{array}{c}\text { max. magnitude error } 1 \mathrm{~dB} \\
\text { max. phase error } 15^{\circ}\end{array}$ & $\begin{array}{c}\text { proposed } \\
\text { model }\end{array}$ & $\begin{array}{c}\text { latest } \\
\text { model }\end{array}$ \\
\hline $100 \mathrm{~km}$, up to $500 \mathrm{~Hz}$ & 14 & 14 \\
\hline $300 \mathrm{~km}$, up to $500 \mathrm{~Hz}$ & 24 & 35 \\
\hline $500 \mathrm{~km}$, up to $500 \mathrm{~Hz}$ & 26 & 70 \\
\hline $\begin{array}{c}\text { max. magnitude error } 0.1 \mathrm{~dB} \\
\text { max. phase error } 1^{\circ}\end{array}$ & $\begin{array}{c}\text { proposed } \\
\text { model }\end{array}$ & $\begin{array}{c}\text { latest } \\
\text { model }\end{array}$ \\
\hline $100 \mathrm{~km}$, up to $500 \mathrm{~Hz}$ & 24 & 50 \\
\hline $300 \mathrm{~km}$, up to $500 \mathrm{~Hz}$ & 26 & 200 \\
\hline $500 \mathrm{~km}$, up to $500 \mathrm{~Hz}$ & 32 & 300 \\
\hline
\end{tabular}

It is expected that the model order of the proposed approach could be further improved for shorter cables and for lower frequency range. The weighting function of the vector fitting would have to be examined thoroughly as well as the distribution of the frequency samples of the frequency response. However, this is out of the scope of this study.

\subsection{Comparison on $70 \mathrm{~km}$ bipolar DC cable}

The proposed method is also compared against the latest state space model on the case of 70k bipolar DC cable. The models are compared in terms of self-impedance as well as the mutual impedance. Analysing mutual impedance is particularly interesting because it investigates ability to represent effect of mutual coupling. The DC cable is composed of two mutually coupled poles because the two poles are physically buried within close proximity. Each pole is composed of conductor, sheath and armor layers.

The latest state space model requires Kron reduction and also modal transformation because there are two coupled poles. Also, the transformation matrix is frequency independent because the bipolar cable is symmetrical with respect to ground. In order to represent frequency range up $1 \mathrm{kHz}$ with a maximum magnitude error of $1 \mathrm{~dB}$ and phase error of $15^{\circ}$, a model of the $212^{\text {th }}$ order is required. The model order is chosen as twice the model order necessary to represent singleconductor DC cables up to $2 \mathrm{kHz}$ (this is provided in [8]). However, the desired accuracy of the self-impedance is achieved up to $1 \mathrm{kHz}$ and the mutual-impedance only up to $500 \mathrm{~Hz}$, approximately.

The proposed method achieves desired accuracy for both the self-impedance and mutual impedance with a slightly lower model order $-200^{\text {th }}$ order. The challenge that arises in this method is to tune vector fitting weighting function for four frequency responses (corresponding to four outputs). This is the reason why the model order is larger then twice the model order necessary to represent single-conductor cable.

Figure 5.4 compares frequency responses of the selfimpedance of two assembled models while Figure 5.5 compares responses of the mutual-impedance. As can be seen from the figures, proposed model achieves higher accuracy and this is particularly pronounced for the mutual impedance. 

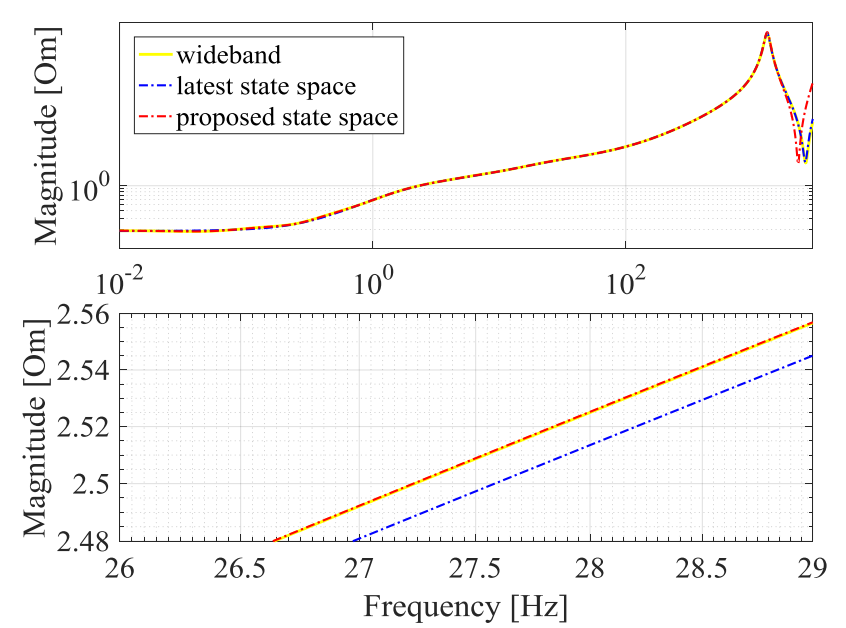

Figure 5.4 Magnitude of the frequency response of the selfimpedance
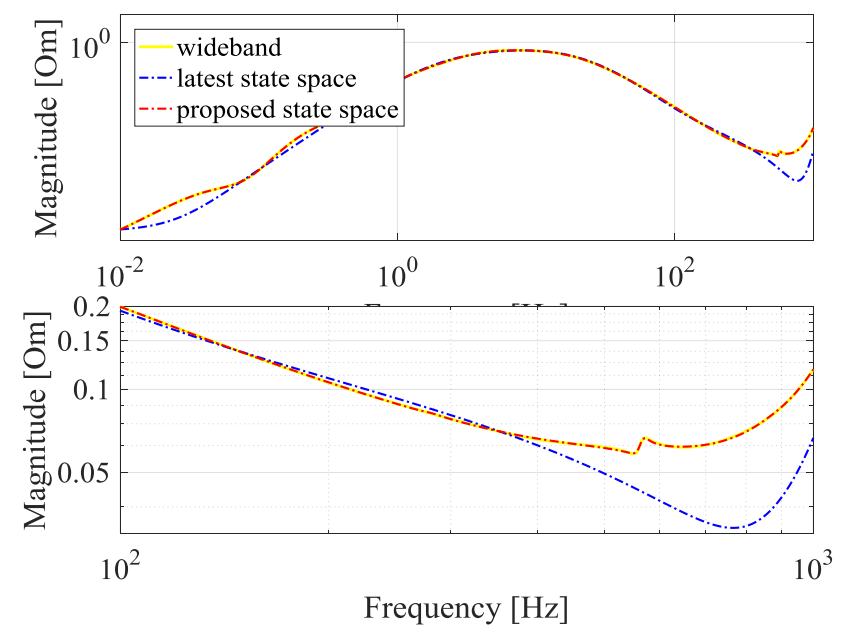

Figure 5.5 Magnitude of the frequency response of the mutual-impedance

\subsection{Application on multi-conductor lines and cables}

The more mutually coupled conductors the transmission systems is composed of, the more difficult it is to minimize model order by tuning weighting function. However, based on the analysis of single-conductor cables in section 5.3 and the bipolar cable in section 5.4, it is reasonably expected that the proposed model is beneficial for longer lines, higher upper frequency limits and for more mutually coupled conductors. The accuracy of the coupling effect is controlled directly using vector fitting as some of the frequency responses translated into state space represent mutual impedance. Proposed model is definitely beneficial for unbalanced lines with pronounced asymmetry because it avoids complex frequency dependent modal transformation. Thorough analysis of the model order and accuracy for multi-conductor lines and cables is a recommendation for further studies.

\section{Conclusions}

The proposed state space model adequately captures the frequency dependent nature of transmission lines and cable. It translates the state of the art time-domain universal line model into state space. This model has shown to have certain advantages over the latest state space model. First of all, DC cables are represented without applying Kron reduction which means improved model fidelity can be achieved. Singleconductor DC cable are represented with a lower model order and higher accuracy for a wide spectre of upper frequency limits and cable lengths. The model order does not depend exponentially on the upper frequency limit which means significant model order reduction when representing wider frequency range. The proposed model has shown to represent bipolar DC cable up $1 \mathrm{kHz}$ with a lower model order and higher accuracy. The effect of mutual coupling is particularly better represented. Multi-conductor lines and cable are represented without applying modal transformation. This overcomes the issue to represent unbalanced systems. Also, the representation of the coupling effect is directly controlled using vector fitting which means desired accuracy can always be achieved.

\section{References}

[1] A. Morched, B. Gustavsen, M. Tartibi. "A universal model for accurate calculation of electromagnetic transients on overhead lines and underground cables", IEEE Trans. on Power Deliv. Vol. 14, No. 3, July 1999.

[2] P. Rault. "Dynamic Modeling and Control of MultiTerminal HVDC Grids", Ph.D. dissertation, Univ. of Lille Nord de France, 2014.

[3] J. Beerten, S. D'Arco, J. A. Suul. "Frequency-dependent cable modelling for small-signal stability analysis of VSC-HVDC systems", IET Gener. Transm. Distrib. vol. 10, no. 6, pp. 1370-1381, 2016.

[4] A. Semlyen, A. Deri. 'Time domain modelling of frequency dependent three-phase transmission line impedance', IEEE Trans. Power Appl. Syst., PAS-104, (6), pp. 1549-1555, 1985.

[5] N. Garcia, E. Acha. 'Transmission line model with frequency dependency and propagation effects: a model order reduction and state-space approach', Proc. IEEE PES GM 2008, Pittsburgh, USA, p. 7, 20-24 July 2008.

[6] B. Gustavsen, A. Semlyen. 'Rational approximation of frequency domain responses by vector fitting', IEEE Trans. Power Deliv., 14, (3), pp. 1052-1061, 1999.

[7] B. Gustavsen, T Noda, J. Naredo. 'Power system transients: parameter determination', (Taylor and Francis Group, 2010), pp. 137-175, Ch. 3

[8] S. D'Arco1, J. A. Suul, J. Beerten. 'Analysis of Accuracy versus Model Order for Frequency-Dependent Pi-model of HVDC Cables', 2016 IEEE 17th Workshop on Control and Modeling for Power Electronics (COMPEL), Pages: 1 - 8, Year: 2016

[9] VFIT3, [Online] Available: https://www.sintef.no/projectweb/vectfit/downloads/ 